Castro, J.; Gabarró, J.; Serna, M. Measuring investment opportunities under uncertainty. A: European Conference on Symbolic and Quantitative Approaches to Reasoning with Uncertainty. "Symbolic and Quantitative Approaches to Reasoning with Uncertainty, 15th European Conference, ECSQARU 2019: Belgrade, Serbia, September 18-20, 2019: proceedings". Berlín: Springer, 2019, p. 481-491.

The final authenticated version is available online at https://doi.org/10.1007/978-3-030-29765-7_40

\title{
Measuring Investment Opportunities under Uncertainty *
}

\author{
Jorge Castro, Joaquim Gabarro, Maria Serna \\ CS Dept. Universitat Politècnica de Catalunya, Barcelona, Spain \\ \{castro, gabarro, mjserna\}@cs.upc.edu
}

\begin{abstract}
In order to make sound economic decisions it is important to measure the possibilities offered by a market in relation to investments. Provided an investment scheme $S=\left\langle r ; R_{1}, \ldots, R_{n}\right\rangle$, where $r$ is a lower bound on the desired investment return and the $R_{i}$ 's are the asset yields, the power to invest measures the capability of the scheme to fulfill requirement $r$. The power to invest is inspired in the Coleman's power of a collectivity to act. We exemplify this approach considering subsets of companies from stock indexes IBEX35 and DAX. We extend the power to invest to investment schemata with imprecise yields. We prove basic relations with the precise yields case and we also show that good monotonicity properties hold. Finally, we propose an analysis, through integrals of the power function, for the case of an unspecific desired return $r$.
\end{abstract}

Keywords. Power to Act, Investment opportunities, Power to Invest, Uncertainty.

\section{Introduction}

The assessment of the economic opportunities offered by a market is an important an unavoidable topic [9]1]. For instance, a start-up will prefer to implement its innovative business idea in a dynamic market rather than in one less promising. In this paper, we consider stock markets and we attempt to provide a quantitative assessment of the intuitive sentence "plentiful of investment opportunities". This issue is a special case of "how to measure the opportunities to do something".

In the framework of cooperative simple games this question was answered precisely by J. Coleman who introduced the power to act [3]. Given a collectivity with $n$ players, the power of such a collectivity to act is:

$$
\text { Power to Act }=\frac{1}{2^{n}} \text { Number of Winning Coalitions }
$$

\footnotetext{
* J. Castro was partially supported by the Spanish Ministry for Economy and Competitiveness (MINECO) under grant (TIN2017- 89244-R) and the recognition 2017 SGR-856 (MACDA) from AGAUR (Generalitat de Catalunya). J. Gabarro and M. Serna were partially supported by MINECO and FEDER funds under grant GRAMM (TIN2017-86727-C2-1-R) and Generalitat de Catalunya, Agéncia de Gestió d'Ajuts Universitaris i de Recerca, under project 2017 SGR 786 (ALniBCOM). M. Serna was also supported by MINECO under grant BGSMath (MDM2014-044).
} 
We adapt this idea to provide information to investors about the degree of freedom of choice. For a portfolio with $n$ assets, we define the power to invest as:

$$
\text { Power to Invest }=\frac{1}{2^{n}-1} \text { Number of Investments with Adequate Expected Return. }
$$

Along the paper, we use the word return of an asset as a synonymous of the yield of this asset. The yield can be defined in different ways depending on the available information or on the investment strategy. The exact meaning of "asset return" in the examples below will be clear from the context. We first introduce the power to invest on portfolios whose assets have precise yield values. We extend later this definition to encompass more realistic scenarios where returns of some assets are uncertain. The power to invest provides a numerical assessment that can be used to compare the investment opportunities in different markets, even in the case that the minimum acceptable return is not precisely defined.

The paper is organized as follows. In Section 2 we introduce the power to invest concept for portfolios whose assets have precise returns and the power function is computed for a couple of stock indexes. Section 3 shows basic properties of the power function. A couple of stylized investment schemata are analyzed. Section 4 extends the power function to assets with imprecise returns. Theorem 1 shows good monotonicity properties for the power even in the case of imprecise returns. Section 5 considers the case where the minimum acceptable return for the investments is unspecific. We show how the integral of the power function can be used to measure investment opportunities in this case. Theorem 2 shows that, under an equiprobable investment schemata and having no information about the minimum acceptable return, the integral of the power function is the average of the asset yields. Finally in Section 6 we summarize the paper and suggest some possible future developments.

\section{Power to Invest}

We consider a set $[n]=\{1, \ldots, n\}$ of assets providing returns $A=\left(R_{1}, \ldots, R_{n}\right)$. We assume that, for each $i$ in $[n], R_{i}>0$. An investment scheme is a pair $S=\langle r ; A\rangle$, where $A$ is the asset return tuple and $r>0$ is the minimum acceptable return for investments. We roughly identify possible investments with non-empty subsets on $[n]$. In order to associate a return to a non-empty subset $I$, it is customary to choose a probability distribution on the assets in $I$. To exemplify our approach, we consider along the paper the uniform distribution which grants a maximal variety and diversification among investments. For an investment $I \subseteq[n]$, the expected return is $E(I)=\frac{1}{|I|} \sum_{i \in I} R_{i}$, where $|I|$ denotes the cardinality of $I$. An investment $I$ is feasible (or acceptable) for $S$ iff $E(I) \geq r$. The set of all feasible investments for scheme $S$ is $F(S)=\{I \mid E(I) \geq r\}$. Therefore, $|F(S)| \leq 2^{n}-1$. The following definition is taken from [2] where the power to invest is briefly analyzed in an strategic setting under the angel-daemon approach [7].

Definition 1. Given an investment scheme $S=\langle r ; A\rangle$ the power to invest is the ratio between the number of feasible investments and the total number of investments. Formally, Power $(S)=\operatorname{Power}(r ; A)=|F(S)| /\left(2^{n}-1\right)$. 


\begin{tabular}{lccc} 
Name & Price & PER & Dividend Yield \\
\hline ACERINOX & 12.10 & 14.38 & $3.73 \%$ \\
DIA & 3.66 & 11,78 & $4.72 \%$ \\
FERROVIAL & 17.23 & 33,90 & $3.17 \%$ \\
IAG (IBERIA) & 7.15 & 6.23 & $4.14 \%$ \\
INDITEX & 25.52 & 20.93 & $3.24 \%$ \\
REPSOL & 13.99 & 9.73 & $6.38 \%$ \\
SANTANDER & 5.34 & 10.64 & $4.03 \%$ \\
SIEMENS GAMESA & 12.90 & $1 ., 82$ & $1.03 \%$ \\
TECNICAS REUNIDAS & 26.34 & 22.84 & $5.52 \%$ \\
TELEFONICA & 8.05 & 11.03 & $4.90 \%$
\end{tabular}

(a) Information for companies in ECO10 index

\begin{tabular}{r|r}
$r$ & Power \\
\hline 0.01 & 1.0 \\
0.02 & 0.999 \\
0.03 & 0.975 \\
0.04 & 0.546 \\
0.05 & 0.030 \\
0.06 & 0.000 \\
0.07 & 0.000
\end{tabular}

(b) Values

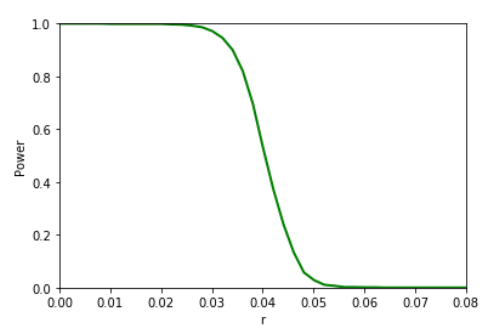

(c) Power $_{E C O 10}(r)$

Fig. 1: Data corresponding to the Companies in the ECO10 Spanish Index. Returns are considered to be the dividend yields.

Sometimes we fix the asset return tuple $A$ taking the minimum acceptable return $r$ as a variable. In such a case the function $F(r ; A)$, as $A$ is fixed, depends only on $r$ and we write $F_{A}(r)$. Similarly, we write in this case $\operatorname{Power}_{A}(r)=\frac{1}{2^{n}-1}\left|F_{A}(r)\right|$.

To get the power to invest for a given market, we need to choose a set of assets. A way to do that is through stock indexes. The IBEX35 [12] is the stock market index of the Bolsa de Madrid and contains the 35 major Spanish stocks. The DAX [10] is a market index consisting of the 25 major German companies trading on the Frankfurt Stock Exchange. The data of the following examples is drawn from the electronic version of the El Economista newspaper [6]. In both cases, we consider the dividend yield or dividend-price ratio i.e., the dividend of a share divided by the price of a share [11], as a proxy of the $R_{i}$ values.

Example 1. Consider the subset of the Spanish IBEX35 companies chosen by the newspaper elEconomista.es [5] used to build the ECO10 index. Data is shown in Figure 1 a The tuple of dividend yields is:

$$
E C O 10=(0.037,0.047,0.031,0.041,0.032,0.063,0.040,0.010,0.055,0.049) .
$$

Figure $1 \mathrm{~b}$ shows some power values and Figure $1 \mathrm{c}$ provides a complete plot. 


\begin{tabular}{lc|lc} 
Name & Dividend Yield & Name & Dividend Yield \\
\hline ADIDAS & $0.00 \%$ & E.ON & $5.21 \%$ \\
ALLIANZ & $4.58 \%$ & FRESENIUS SE & $1.20 \%$ \\
BASF SE & $3.77 \%$ & HENKEL & $0.00 \%$ \\
BAYER & $2.96 \%$ & MERCK KGAA & $0.00 \%$ \\
BMW & $4.52 \%$ & MUNICH RE & $4.87 \%$ \\
COMMERZBANK & $0.00 \%$ & PROSIEBEN SAT.1 N & $0.00 \%$ \\
CONTINENTAL & $0.00 \%$ & RWE & $6.96 \%$ \\
DAIMLER & $5.44 \%$ & SAP & $1.61 \%$ \\
DEUTSCHE & $1.92 \%$ & SIEMENS & $3.60 \%$ \\
DEUTSCHE BOERSE & $6.04 \%$ & THYSSENKRUPP & $0.00 \%$ \\
DEUTSCHE LUFTHANSA & $0.00 \%$ & VOLKSWAGEN VORZ & $0.00 \%$ \\
DEUTSCHE POST & $3.23 \%$ & VONOVIA N & $0.00 \%$ \\
DEUTSCHE TELEKOM & $5.29 \%$ & &
\end{tabular}

(a) Information for companies in DAX

\begin{tabular}{r|r}
$r$ & Power \\
\hline 0.01 & 1.0 \\
0.02 & 0.999 \\
0.03 & 0.988 \\
0.04 & 0.622 \\
0.05 & 0.036 \\
0.06 & 0.000 \\
0.07 & 0.000
\end{tabular}

(b) Values

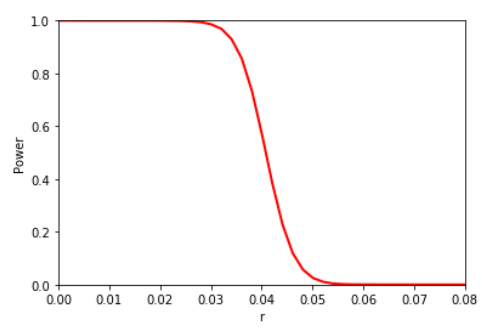

(c) $\operatorname{Power}_{D A X}(r)$

Fig. 2: Data corresponding to the companies belonging the DAX German Index. We consider as returns the dividend yields.

Example 2. Data of the German DAX is drawn from [4]. Yields are given in Figure 2a. To compute the power we only consider the non-zero yield assets. So, here the number of assets reduces to $n=15$.

$$
\begin{aligned}
D A X= & (0.0458,0.0377,0.0296,0.0452,0.0544,0.0192,0.0604 \\
& 0.0323,0.0529,0.0521,0.0120,0.0487,0.0696,0.0161,0.0360) .
\end{aligned}
$$

Figure $2 \mathrm{~b}$ gives some power values and Figure $2 \mathrm{c}$ shows the function plot.

Given a market, represented by $A$, the power to invest provides a measure of the investment opportunities. We propose to use $\operatorname{Power}_{A}(r)$ to compare markets. We prefer the market with the highest power to invest. The preference may depend on the specific value of $r$, as we illustrate in the following example.

Example 3. The power to invest, for ECO10 and DAX, seems quite similar (see Figure 3a). However, looking in more detail, we get additional insight. For $r \in(0,0.0426]$, the $D A X$ is better. For $r \geq 0.0427, E C O 10$ is the best option (see Figure $3 \mathrm{~b}$ ). 


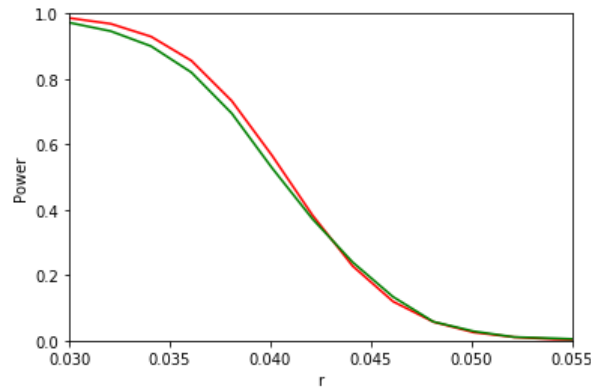

(a) Powers of ECO10 and DAX

\begin{tabular}{l||c|c|c}
$r$ & Power $_{E C O 10}$ & & Power $_{\text {DAX }}$ \\
\hline 0.042 & 0.3831 & $<$ & 0.3914 \\
0.0425 & 0.3431 & $<$ & 0.3481 \\
0.0426 & 0.3401 & $<$ & 0.3402 \\
\hline 0.0427 & 0.3294 & $>$ & 0.3315 \\
0.0428 & 0.3245 & $>$ & 0.3239 \\
0.043 & 0.3167 & $>$ & 0.3078 \\
0.05 & 0.0303 & $>$ & 0.0263
\end{tabular}

(b) Numerical comparison on powers

Fig. 3: Powers corresponding to ECO10 and DAX. Initially DAX outperforms ECO10 in relation to the power. At some point in $[0.0426,0.0427]$ the relation is reversed.

\section{Stylized properties}

We start showing several basic properties of the power function including some elementary monotonicity relationships. Let $A=\left(R_{1}, \ldots, R_{n}\right)$ and $\hat{A}=\left(\hat{R}_{1}, \ldots, \hat{R}_{n}\right)$ be two return tuples. As usual we say that $A \leq \hat{A}$ iff for each $i$ in $[n]$ it is $R_{i} \leq \hat{R}_{i}$.

Lemma 1. Let $A=\left(R_{1}, \ldots, R_{n}\right)$ and $\hat{A}=\left(\hat{R}_{1}, \ldots, \hat{R}_{n}\right)$ be two return tuples. The following holds

- $0 \leq \operatorname{Power}_{A}(r) \leq 1$, for $r>0$.

- $\operatorname{Power}_{A}(r)=1$, for $r \leq \min _{i \in[n]} R_{i}$ and $\operatorname{Power}_{A}(r)=0$, for $r>\max _{i \in[n]} R_{i}$.

- $\operatorname{Power}_{A}(r)$ is decreasing, i.e., for $r \leq r^{\prime}, \operatorname{Power}_{A}(r) \geq \operatorname{Power}_{A}\left(r^{\prime}\right)$.

- Power $(r ; A)$ is increasing with respect to $A$ i.e., if $A \leq \hat{A}$, Power $(r ; A) \leq \operatorname{Power}(r ; \hat{A})$.

Let us consider some stylized investment schemata. First, we consider a case where all the assets have the same return. We denote $n$ identical returns $R$ by $n: R$. So, we write the scheme $S=\langle r ; R, \ldots, R\rangle$ in a shorter way as $S=\langle r ; n: R\rangle$.

Lemma 2. Let $S=\langle r ; n: R\rangle$. It holds $\operatorname{Power}(S)=1$ when $r \leq R$ and 0 otherwise.

For instance, Figure 4a plots function $\operatorname{Power}_{A}(r)$ for $S=\langle r ; A\rangle=\langle r ; 0.05\rangle$.

Lemma 3. Let $S$ be a scheme with two different returns, $S=\left\langle r, n_{1}: R_{1}, n_{2}: R_{2}\right\rangle$. Assume w.l.o.g that $R_{1} \leq R_{2}$. When $r \leq R_{1}$ the power is 1 and when $r>R_{2}$ the power is 0 . When $R_{1} \leq r \leq R_{2}$, we have

$$
\operatorname{Power}(S)=\frac{1}{2^{n_{1}+n_{2}}-1} \sum_{\left(k_{1}, k_{2}\right) \in P_{S}}\left(\begin{array}{l}
n_{1} \\
k_{1}
\end{array}\right)\left(\begin{array}{l}
n_{2} \\
k_{2}
\end{array}\right)
$$

where $P_{S}=\left\{\left(k_{1}, k_{2}\right) \mid\left(k_{1}+k_{2}\right) r \leq k_{1} R_{1}+k_{2} R_{2}, k_{1} \leq n_{1}, k_{2} \leq n_{2}, k_{1}+k_{2}>0\right\}$.

Consider the return tuples $A_{1}=(1: 0.05,1: 0.15), A_{2}=(2: 0.05,1: 0.15)$ and $A_{3}=$ $(4: 0.05,7: 0.15)$. The respective power plots are given in Figures $4 \mathrm{~b}$, $4 \mathrm{c}$ and $4 \mathrm{~d}$. All plots have stepwise shapes. This type of shape also holds in the general case. 


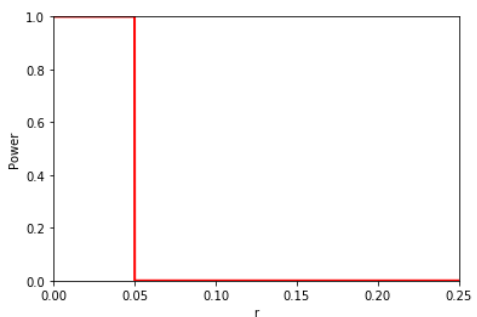

(a) $A_{1}=(1: 0.05)$

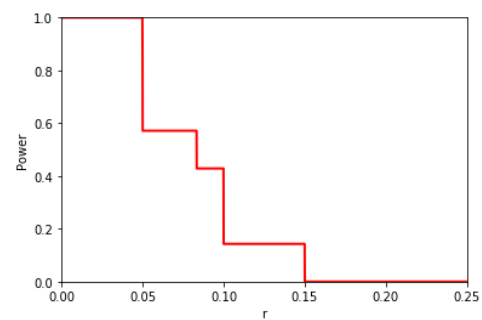

(c) $A_{3}=(2: 0.05,1: 0.15)$

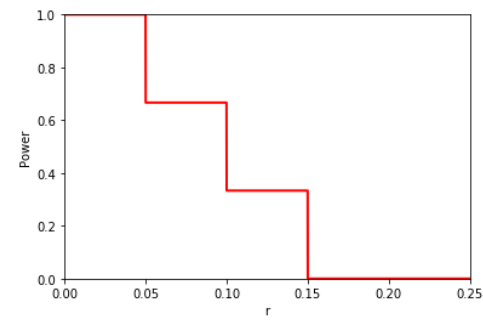

(b) $A_{2}=(1: 0.05,1: 0.15)$

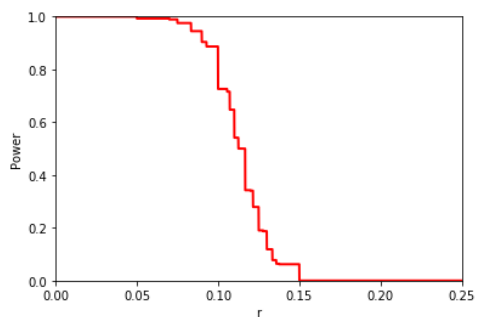

(d) $A_{4}=(4: 0.05,7: 0.15)$

Fig. 4: Function $\operatorname{Power}\left(r, n_{1}: 0.05, n_{2}: 0.15\right)$ for several values of $n_{1}$ and $n_{2}$.

Lemma 4. Let $A=\left(R_{1}, \ldots, R_{n}\right)$ be an asset return tuple. The function $\operatorname{Power}_{A}(r)$ is a non-increasing stepwise function having a finite number of discontinuity points in the interval $[x, y]$ where $x=\min _{i \in[n]} R_{i}$ and $y=\max _{i \in[n]} R_{i}$. Furthermore, $\operatorname{Power}_{A}(r)=1$, for $r \in[0, x]$, and $\operatorname{Power}_{A}(r)=0$, for $r \in(y,+\infty)$.

Proof. Given $A=\left(R_{1}, \ldots, R_{n}\right)$, we consider the function $f_{I}(r): \mathcal{P}([n]) \times \mathbb{R}^{+} \rightarrow$ $\{0,1\}$ defined to be 1 when $\frac{1}{|I|} \sum_{i \in I} R_{i} \geq r$ and 0 otherwise. Observe that, for each $I \neq \emptyset, f_{I}(r)$ is stepwise and has an unique discontinuity point. The result follows from the equality $\operatorname{Power}_{A}(r)=\left(\sum_{I \subseteq[n]} f_{I}(r)\right) /\left(2^{n}-1\right)$.

\section{Imprecise Returns}

We consider a simple model of imprecision where each asset yield is an unspecific value in a closed interval.

Definition 2. A tuple of imprecise returns $A$ is formed by $\left(R_{1}, \ldots, R_{n}\right)$ where each $R_{i}$ is a closed interval $\left[R_{i}^{-}, R_{i}^{+}\right]$. Return of asset $i$ is some unspecific value in $R_{i}$. An investment scheme on imprecise returns is $S=\langle r ; A\rangle$ where tuple $A$ is imprecise.

Observe that any precise return tuple $\left(R_{1}, \ldots, R_{n}\right)$ can be understood as an imprecise one by defining $R_{i}=\left[R_{i}, R_{i}\right]$. On the other hand, an investment scheme on imprecise returns $S=\left\langle r ; R_{1}, \ldots, R_{n}\right\rangle$ defines two natural extreme investment schemata on precise ones: $S^{-}=\left\langle r ; R_{1}^{-}, \ldots, R_{n}^{-}\right\rangle$and $S^{+}=\left\langle r ; R_{1}^{+}, \ldots, R_{n}^{+}\right\rangle$. For imprecise return 
tuple $A=\left(R_{1}, \ldots, R_{n}\right)$ we also consider the two extreme precise return tuples $A^{-}$ and $A^{+}$. Under equiprobable weights, for $I \subseteq[n]$, the expected returns under those extreme cases are $E^{-}(I)=\frac{1}{|I|} \sum_{i \in I} R_{i}^{-}$and $E^{+}(I)=\frac{1}{|I|} \sum_{i \in I} R_{i}^{+}$. We also consider $F\left(S^{+}\right)=\left\{I \mid E^{+}(I) \geq r\right\}$ and $F\left(S^{-}\right)=\left\{I \mid E^{-}(I) \geq r\right\}$. The following monotonicity properties hold.

Lemma 5. Given a tuple of imprecise returns $A=\left(R_{1}, \ldots, R_{n}\right)$ where, for $i \in[n]$, $R_{i}=\left[R_{i}^{-}, R_{i}^{+}\right]$, it holds $E^{-}(I) \leq E^{+}(I)$ and $F\left(S^{-}\right) \subseteq F\left(S^{+}\right)$.

We extend the power function to the case of imprecise returns by considering the following intuition. Given scheme $S$ on imprecise returns in the previous paragraph, investment $I \in F\left(S^{-}\right)$contributes $1 /\left(2^{n}-1\right)$ to the power sum. This is because for any returns choice in the imprecise intervals, investment $I$ will be feasible. On the contrary, when $I \notin F\left(S^{+}\right)$, whatever the yield values are the investment is not feasible and the contribution is zero. Finally, for the intermediate case where $I \in F\left(S^{+}\right) \backslash F\left(S^{-}\right)$ the contribution of $I$ can be considered to be $\left(E^{+}(I)-r\right) /\left(E^{+}(I)-E^{-}(I)\right)$ times $1 /\left(2^{n}-1\right)$. Summing up, we provide the following extension of the power definition.

Definition 3. Let $S=\left\langle r ; R_{1}, \ldots, R_{n}\right\rangle$ be an investment scheme on imprecise returns where for each $i \in[n]$ interval $R_{i}$ is $\left[R_{i}^{-}, R_{i}^{+}\right]$. The power to invest is defined as

$$
\operatorname{Power}(S)=\frac{1}{2^{n}-1} \sum_{I \in F\left(S^{+}\right)} \frac{E^{+}(I)-\max \left\{r, E^{-}(I)\right\}}{E^{+}(I)-E^{-}(I)}
$$

Here the expression $\left(E^{+}(I)-E^{-}(I)\right) /\left(E^{+}(I)-E^{-}(I)\right)$ is considered to be 1 when $E^{+}(I)=E^{-}(I)$ so that Power for imprecise returns extends Power for precise ones.

We first analyze some basic properties of Power. As one can expect the power of an investment scheme on imprecise returns lies between the power of its two extreme investment schemata.

Lemma 6. Given an imprecise scheme $S$, it holds $\operatorname{Power}\left(S^{-}\right) \leq \operatorname{Power}(S) \leq \operatorname{Power}\left(S^{+}\right)$.

Example 4. Consider the scheme $S=\langle r ; n: R\rangle$ given in Lemma 2. We assume a volatility $\sigma$ on $R$ such that $R>\sigma$. We model this volatility into imprecision by considering $R^{\prime}=[R-\sigma, R+\sigma]$ and defining the imprecise return tuple $A=\left(n: R^{\prime}\right)$. In this case we have $A^{-}=(n: R-\sigma)$ and $A^{+}=(n: R+\sigma)$. From Lemma 2, we have,

$$
\text { Power }_{A^{-}}(r)=\left\{\begin{array}{ll}
1 & \text { if } r \leq R-\sigma \\
0 & \text { otherwise }
\end{array} \text { Power }_{A^{+}}(r)=\left\{\begin{array}{lc}
1 & \text { if } r \leq R+\sigma \\
0 & \text { otherwise }
\end{array}\right.\right.
$$

For an investment $I \subseteq[n], E^{-}(I)=R-\sigma$ and $E^{+}(I)=R+\sigma$. Therefore,

$$
\operatorname{Power}_{A}(r)= \begin{cases}1 & \text { if } r \leq R-\sigma \\ \frac{R+\sigma-r}{2 \sigma} & \text { if } R-\sigma<r \leq R+\sigma \\ 0 & \text { if } R+\sigma<r\end{cases}
$$

Power $_{A}$ is a "smooth average" between Power $A^{-}$and Power $A^{+}$. See Figure 5 a 


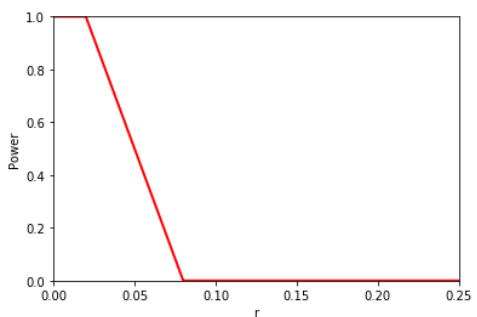

(a) $A_{1}=(1:[0.02,0.08])$

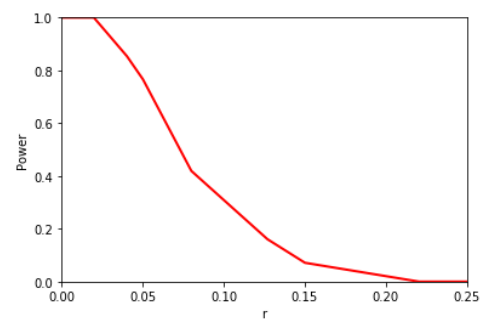

(c) $A_{3}=(2:[0.02,0.08], 1:[0.08,0.22])$

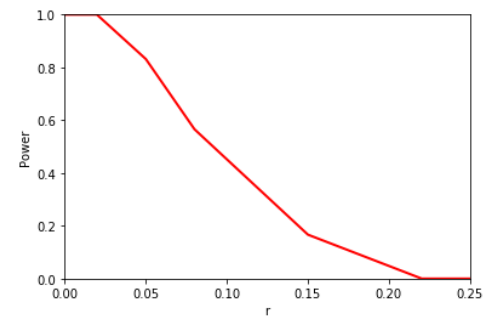

(b) $A_{2}=(1:[0.02,0.08], 1:[0.08,0.22])$

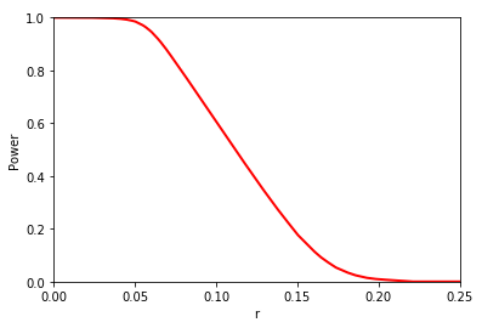

(d) $A_{4}=(4:[0.02,0.08], 7:[0.08,0.22])$

Fig. 5: Power $\left(r, n_{1}:[0.02,0.08], n_{2}:[0.08,0.22]\right)$ for several $n_{1}$ and $n_{2}$ values.

Example 5. Consider the investment scheme $S=\left\langle r ; n_{1}: R_{1}, n_{2}: R_{2}\right\rangle$ given in Lemma 3 , where $R_{1}<R_{2}$. We assume volatilities $\sigma_{1}$ and $\sigma_{2}$ on, respectively, returns $R_{1}$ and $R_{2}$ such that $R_{1}>\sigma_{1}$ and $R_{2}>\sigma_{2}$. We model this volatility into imprecision by considering $R_{1}^{\prime}=\left[R_{1}-\sigma_{1}, R_{1}+\sigma_{1}\right]$ and $R_{2}^{\prime}=\left[R_{2}-\sigma_{2}, R_{2}+\sigma_{2}\right]$, and defining the imprecise return tuple $A=\left(n_{1}: R_{1}^{\prime}, n_{2}: R_{2}^{\prime}\right)$. Figure 5 shows the plot for $R_{1}=0.05, R_{2}=0.15$, $\sigma_{1}=0.03$ and $\sigma_{2}=0.07$ (so, $R_{1}^{\prime}=[0.02,0.08]$ and $R_{2}^{\prime}=[0.08,0.22]$ ) and several values of $n_{1}$ and $n_{2}$.

We introduce now a partial order among imprecise returns

Definition 4. We say that the imprecise return tuple $\hat{A}=\left(\hat{R}_{1}, \ldots, \hat{R}_{n}\right)$ improves imprecise tuple $A=\left(R_{1}, \ldots, R_{n}\right)$, denoted as $A \sqsubseteq \hat{A}$, when for each $i \in[n]$ it is $R_{i}^{-} \leq \hat{R}_{i}^{-}$and $R_{i}^{+} \leq \hat{R}_{i}^{+}$. When $S=\langle r ; A\rangle$ and $\hat{S}=\langle r ; \hat{A}\rangle$ and $A \sqsubseteq \hat{A}$ we say that $\hat{S}$ improves the investment scheme $S$. Formally we write $S \sqsubseteq \hat{S}$.

Our next results shows that Power is monotonic with respect to this partial order.

Theorem 1. Let $S$ and $\hat{S}$ be two investment schemata on imprecise returns. If $S \sqsubseteq \hat{S}$ then Power $(S) \leq \operatorname{Power}(\hat{S})$.

Proof. Assume that $S=\langle r ; A\rangle$ and $\hat{S}=\langle r ; \hat{A}\rangle$. As $A \sqsubseteq \hat{A}$, we have that $F\left(S^{+}\right) \subseteq$ $F\left(\hat{S}^{+}\right)$and $F\left(S^{-}\right) \subseteq F\left(\hat{S}^{-}\right)$. For an investment $S$ and a subset $I \subseteq[n]$ we consider 
the function

$$
f_{S, I}(r)= \begin{cases}1 & \text { if } r \leq E^{-}(I) \\ \frac{E^{+}(I)-r}{E^{+}(I)-E^{-}(I)} & \text { if } E^{-}(I)<r \leq E^{+}(I) \\ 0 & \text { if } E^{+}(I)<r .\end{cases}
$$

In the following, with an abuse of notation, we use $\hat{E}$ to denote the expected returns under returns $\hat{A}$. As $A \sqsubseteq \hat{A}$, we have that $E^{-}(I) \leq \hat{E}^{-}(I)$ and $E^{+}(I) \leq \hat{E}^{+}(I)$. We have to study the relationship among $f_{S, I}$ and $f_{\hat{S}, I}$ depending on the value of $r$. When $r \leq E^{-}(I), f_{S, I}(r)=f_{\hat{S}, I}(r)=1$. When $r>\hat{E}^{+}(I), f_{S, I}(r)=f_{\hat{S}, I}(r)=0$. Now we have to consider two cases.

Case 1: $E^{+}(I)<\hat{E}^{-}(I)$. In this case we have three relevant intervals to consider for $r$. When $r \in\left[E^{-}(I), E^{+}(I)\right], f_{S, I}(r) \leq 1$ and $f_{\hat{S}, I}(r)=1$. When $r \in\left(E^{+}(I), \hat{E}^{-}(I)\right]$, $f_{S, I}(r)=0$ and $f_{\hat{S}, I}(r)=1$. Finally, when $r \in\left(\hat{E}^{-}(I), \hat{E}^{+}(I)\right], f_{S, I}(r)=0$ and $0 \leq f_{\hat{S}, I}(r) \leq 1$. We conclude that $f_{S, I} \leq f_{\hat{S}, I}$.

Case 2: $\hat{E}^{-}(I) \leq E^{+}(I)$. Again we have to consider three intervals for $r$. When $r \in$ $\left[E^{-}(I), \hat{E}^{-}(I)\right], f_{S, I}(r) \leq 1$ and $f_{\hat{S}, I}(r)=1$. When $r \in\left(E^{+}(I), \hat{E}^{+}(I)\right], f_{S, I}(r)=$ 0 and $0 \leq f_{\hat{S}, I}(r) \leq 1$. Finally, when $r \in\left[\hat{E}^{-}(I), E^{+}(I)\right], f_{S, I}(r)=\left(E^{+}(I)-\right.$ $r) /\left(E^{+}(I)-E^{-}(I)\right)$ and $f_{\hat{S}, I}(r)=\left(\hat{E}^{+}(I)-r\right) /\left(\hat{E}^{+}(I)-\hat{E}^{-}(I)\right)$. Introducing the non-negative values $a=\hat{E}^{-}(I)-E^{-}(I), b=r-\hat{E}^{-}(I), c=E^{+}(I)-r$ and $d=$ $\hat{E}^{+}(I)-E^{+}(I)$, we can write $f_{S, I}(r)=c /(c+a+b)$ and $f_{\hat{S}, I}(r)=(c+d) /(c+d+b)$. From this expression, after some algebraic calculation, it follows that $f_{S, I} \leq f_{\hat{S}, I}$. As it holds that $\left.\operatorname{Power}(S)=\left(\sum_{I \subseteq[n]} f(S, I)\right)\right) /\left(2^{n}-1\right)$ the claim follows.

For instance, given imprecise return tuples $A=(2:[0.02,0.08], 1:[0.08,0.22])$ and $\hat{A}=(2:[0.02,0.09], 1:[0.085,0.23])$ it holds that $A \sqsubseteq \hat{A}$ and thus, by Theorem 1 $\operatorname{Power}_{\hat{A}}(r)$ is greater or equal than $\operatorname{Power}_{A}(r)$ for any value of $r$. Independently of the minimum acceptable return, we can conclude that the investement scheme defined on $\hat{A}$ will offer more investment opportunities.

\section{Power under uncertain minimum return}

In this section we offer a quantitative assessment tool of opportunities for the case of an investor attempting to ensure an unspecific acceptable return in a predefined interval.

A natural way to provide a valuation on an interval of acceptable returns is to consider the integral of the power function on such interval. The larger the integral value is, more business opportunities the market will offer on average, although it may have a worse performance for some return values in the uncertainty interval.

We introduce a bit of notation. For a tuple of imprecise yields $A$ and two real numbers $0 \leq x \leq y$ defining the interval of acceptable investment returns, let us define:

$$
\text { IntegralPower }_{A}(x, y)=\int_{x}^{y} \operatorname{Power}_{A}(r) d r \text {. }
$$

We analyze the case of having no information at all about the acceptable return. We do the valuation of this case with the IntegralPower $(0,+\infty)$ value, that will be denoted in short by IntegralPower(0). 
Lemma 7. Given a tuple of imprecise returns $A=\left(R_{1}, \ldots, R_{n}\right)$

$$
\text { IntegralPower } A_{A}(0)=\frac{\text { IntegralPower }_{A^{-}}(0)+\text { IntegralPower }_{A^{+}}(0)}{2} .
$$

Using the fact that inside of each investment we are using the uniform distribution we can derive another expression for the total integral value.

Theorem 2. For a tuple of imprecise returns $A=\left(R_{1}, \ldots, R_{n}\right)$,

$$
\text { IntegralPower }_{A}(0)=\frac{1}{n} \sum_{i=1}^{n} \frac{R_{i}^{-}+R_{i}^{+}}{2} .
$$

For the case of precise returns the following holds.

Corollary 1. Given a tuple of precise returns $A=\left(R_{1}, \ldots, R_{n}\right)$, IntegralPower $_{A}(0)=\left(\sum_{i=1}^{n} R_{i}\right) / n$.

Example 6. We consider the tuple of asset returns corresponding to ECO10 and DAX given in Examples 1 and 2. From Corollary 1. IntegralPower ${ }_{E C O 10}(0)=0.0405$ and IntegralPower $\operatorname{DAX}(0)=0.0408$. We can interpret these values as follows. For the ECO10 case, choosing an investment uniformly at random is expected - as averagea return of value $4.05 \%$. In contrast, we expect a bit greater return (4.08\%) for a random investment on the $D A X$ index.

\section{Conclusion and open problems}

We have developed different facets of the power to invest. We have seen that this notion has both interesting mathematical properties and potential applicability. The power to invest has the flexibility to be adapted to deal with uncertainty in two dimensions. Uncertainty can arise on the asset yields or on the minimum acceptable investment return.

We have developed the equiprobable approach, but there are other possibilities. Harry Markowitz introduced the mean-variance approach [8]. Given $R_{1}, \ldots R_{n}$ as a part of and investment scheme $S=\left\langle r, R_{1}, \ldots R_{n}\right\rangle$, a portfolio $w=\left(w_{1}, \ldots, w_{n}\right) \in$ $\Delta_{n}$ provides a probability distribution on $[n]$, i.e., positive weights with $\sum_{i \in[w]} w_{i}=1$. The expected return (the mean) is $E(S, w)=\sum_{i \in[n]} w_{i} R_{i}$. For investment $I \subseteq[n]$ the expected return equals to $E(I)=\sum_{i \in I} w_{i} R_{i} / \sum_{i \in I} w_{i}$. From these values a power function $\operatorname{Power}(S, w)$ can be also defined. We are working towards analyzing properties of this function for generic portfolios.

In [2] we consider the angel-daemon approach [7] to investment schemata. The approach tries to tune cases in-between the worst and the best scenarios and analyses them through game theory. The relation between angel-daemon games and the current approach requires further analysis.

\section{References}

1. Adam, T., Goyal, V.: The investment opportunity set and its proxy variables. The Journal of Financial Research 31, 41-63 (2008), https: / /doi.org/10.1111/j.1475-6803. $2008.00231 . x$ 
2. Castro, J., Gabarro, J., Serna, M.: Power to invest. In: Sarabia, J., Prieto, F., M.Guillén (eds.) Contributions to Risk Analysis: RISK 2018. Cuadernos de la Fundación, vol. 223, pp. 135142. Insurance Sciences Institute of Fundación MAPFRE (2018)

3. Coleman, J.: Control of collectivities and the power of a collectivity to act. In: Lieberman, B. (ed.) Social Choice, pp. 269-300. Gordon and Breach (1971), reedited in Routledge Revivals, 2011

4. elEconomista.es: Dax30, rentabilidad/dividendo, http://www.eleconomista.es/ indice/DAX-30/resumen/Rentabilidad-Dividendo

5. elEconomista.es: Eco10, rentabilidad/dividendo, http://www.eleconomista.es/ indice/ECO10/resumen/Rentabilidad-Dividendo

6. elEconomista.es: Portada, http://www. eleconomista.es

7. Gabarro, J., Serna, M., Stewart, A.: Analysing web-orchestrations under stress using uncertainty profiles. The Computer Journal 57(11), 1591-1615 (2014), https://doi.org/ $10.1093 / \mathrm{comjnl} / \mathrm{bxt} 063$

8. Markowitz, H.: Portafolio Selection. John Wiley, London (1959)

9. Skinner, D.: The investment opportunity set and accounting procedure choice: Preliminary evidence. Journal of Accounting and Economics 16, 407-445 (1993), https://doi. org/10.1016/0165-4101(93) 90034-D

10. WikipediA: Dax,https://en.wikipedia.org/wiki/DAX

11. WikipediA: Divident yield, https://en.wikipedia.org/wiki/Dividend_ yield

12. WikipediA: Ibex35,https://en.wikipedia.org/wiki/IBEX_35 